\title{
Soft silicone rubber in phononic structures: Correct elastic moduli
}

\author{
Tim Still, ${ }^{1, *}$ M. Oudich, ${ }^{2, \dagger}$ G. K. Auerhammer, ${ }^{1}$ D. Vlassopoulos, ${ }^{3}$ B. Djafari-Rouhani, ${ }^{2, \dagger}$ G. Fytas, ${ }^{1,3, \S}$ and P. Sheng ${ }^{4}$ \\ ${ }^{1}$ Max Planck Institute for Polymer Research, Ackermannweg 10, 55128 Mainz, Germany \\ ${ }^{2}$ Institut d'Electronique de Microélectronique et de Nanotechnologie UMR CNRS 8520, Université de Lille 1, Sciences et Technologies, \\ 59652 Villeneuve d'Ascq, France \\ ${ }^{3}$ Department of Materials Science \& Technology, University of Crete and F.O.R.T.H./IESL, P.O. Box 1527, 71110 Heraklion, Greece \\ ${ }^{4}$ Department of Physics, Hong Kong University of Science and Technology, Clear Water Bay, Kawloon, Hong Kong, China
}

(Received 27 May 2013; published 3 September 2013)

\begin{abstract}
We report on a combination of experiments to determine the elastic moduli of a soft poly (dimethylsiloxane) rubber that was utilized in a smart experiment on resonant phononic modes [Liu et al., Science 289, 1734 (2000)] and whose reported moduli became widely used as a model system in theoretical calculations of phononic materials. We found that the most peculiar hallmark of these values, an extremely low longitudinal sound velocity, is not supported by our experiments. Anyhow, performing theoretical band structure calculations, we can reproduce the surprising experimental findings of Liu et al. even utilizing the correct mechanical parameters. Thus, the physical conclusions derived in the theoretical works do not require the use of an extremely low longitudinal velocity, but can be reproduced assuming only a low value of the shear modulus, in agreement with our experiments.
\end{abstract}

DOI: 10.1103/PhysRevB.88.094102 PACS number(s): 43.40.+s, 62.20.D-, 63.20.D-, 63.20.Pw

\section{INTRODUCTION}

Phononic structures exhibit periodic changes in their elastic properties that allow them to mold the propagation of sound waves or phonons and, thus, to control the propagation of sound or heat. ${ }^{1-3}$ In recent years, some effort has been taken to expand the concept of phononic effects beyond simple Bragg interference, ${ }^{3-5}$ including the clever design of hybrid structures consisting of materials of very different elastic properties.

Silicone rubber, a weakly cross-linked poly(dimethylsiloxane) (PDMS) material, is often used as a soft component in such phononic hybrid structures. ${ }^{4,6,7}$ However, the reported longitudinal modulus takes an extremely low value of $M=680 \mathrm{kPa},{ }^{8}$ in contrast to the density, which assumes a value typical for dense polymers, $\rho=1300 \mathrm{~kg} / \mathrm{m}^{3}$. These values have been used not only to represent experimental phononic band diagrams ${ }^{4,6,7}$ but also to perform theoretical calculations of phononic structures. ${ }^{9-12}$

Owing to the presence of cross links, this silicone rubber is reported to possess a small but finite shear modulus $G=40 \mathrm{kPa} .{ }^{8}$ Hence, the longitudinal sound velocity $c_{l}=$ $(M / \rho)^{1 / 2} \approx 23 \mathrm{~m} / \mathrm{s}$ is beyond any physically meaningful value in polymer science; for a low molecular weight (i.e., liquid) PDMS, $c_{l}=1050 \mathrm{~m} / \mathrm{s}$ and $G$ is zero because shear waves cannot be supported. ${ }^{5}$ The shear modulus of bulk polymers, on the other hand, can assume any value from zero (Newtonian liquids) up to GPa (elastic solids).

This particular property offers a new way to build up reduced size systems based on this kind of phononic crystal, called acoustic metamaterial, for low-frequency applications such as noise insulation. In fact, the dynamic effective mass density of this metamaterial displays a rapid variation in the vicinity of the gap and may become negative over a certain frequency range. The question is whether this behavior requires very low values for both $M$ and $G$, while the reported very low value of $M$ seems unphysical, or if a low $G$ alone was sufficient to explain the experimental findings.
To this extent, we have measured the elastic moduli of the same soft rubber used in Ref. 4 employing Brillouin light scattering, ultrasonic transmission, rheometry, and nanoindentation techniques; we find, in particular, a significantly higher longitudinal modulus. We then recalculated the band diagram of the locally resonant material using the correct elastic moduli. Quantitatively, the agreement between the theoretical representation and the experimental band gap becomes somewhat less satisfactory when we use the correct longitudinal modulus, but we still find the qualitative overall behavior found in the experiments of Ref. 4 .

\section{RESULTS AND DISCUSSION}

\section{A. Experimental results}

For the sake of clarity, we discuss our results mostly in terms of longitudinal modulus, $M=\rho c_{l}^{2}$, and shear modulus, $G$. The relations between $M, G$, and other elastic parameters (including the Lamé coefficient $\lambda$ that is used in Ref. 4, and Young's modulus $E$ that is obtained directly in nanoindentation experiments) are summarized in Table I for convenience.

We first verified that the measured and reported values of density are in excellent agreement performing a simple Archimedes' bathtub-like measurement $\left(\rho \approx 1300 \mathrm{~kg} / \mathrm{m}^{3}\right)$. Noteworthily, the density value falls in the upper range of densities of bulk polymers. This allows us to determine $M$ directly by measuring sound velocities.

Brillouin light scattering. We utilized Brillouin light scattering (BLS) spectroscopy to measure the acoustic longitudinal phonon. Due to the low transmission of the opaque soft silicon rubber we used a thin slice and record frequency-dependent spectra at different scattering wave vectors $q$ parallel to the film [Fig. 1(a)]; the presence of multiple light scattering from this industrial product is at the origin of the broad BLS spectra due to unavoidable distribution of the $q$ values. In this transmission geometry, ${ }^{13} q$ is independent of the refractive index and readily 
TABLE I. Elastic properties expressed in terms of longitudinal modulus $M=\rho c_{l}^{2}$ and shear modulus $G=\rho c_{t}^{2}$ and useful alternative relations.

\begin{tabular}{lc}
\hline \hline Young's modulus & $E=\frac{G(3 M-4 G)}{M-G}=2 G(1+v)$ \\
Lamé coefficient & $\lambda=M-2 G=\frac{E v}{(1+v)(1-2 v)}$ \\
Bulk modulus & $K=M-\frac{4}{3} G=\frac{E}{3(1-2 v)}$ \\
Poisson's ratio & $v=\frac{M-2 G}{2 M-2 G}=\frac{E}{2 G}-1$ \\
\hline \hline
\end{tabular}

tuned by the scattering angle. As shown in Fig. 1(b), the observed linear dispersion of the longitudinal phonons leads to $c_{l}=2 \pi f / q=1070 \pm 100 \mathrm{~m} / \mathrm{s}$ at $20{ }^{\circ} \mathrm{C}$, where $f$ is the phonon frequency obtained from fits to the spectra in Fig. 1(a). Our longitudinal sound velocity is in good agreement with tabulated values $\left(c_{l} \approx 980 \mathrm{~m} / \mathrm{s}\right) .{ }^{14}$

It should be noted that, in principle, BLS can be utilized to measure the transverse sound velocity and hence the shear modulus, but that the required depolarized scattering is very weak in some samples. In the present sample, we were not able to measure any reliable depolarized signal, in agreement with the already very broad polarized spectra shown in Fig. 1(a).

Ultrasonic transmission. The possibility of a frequency dispersion of $c_{l}(\omega)$ is excluded on the account of the very fast relaxation times of PDMS (given its low glass transition temperature of about $-100{ }^{\circ} \mathrm{C}$ ) and the usually weak dispersion; ${ }^{15}$ the glass transition $\alpha$-relaxation time becomes of the order of $1 / 2 \pi f$ at $T \approx T_{g}+150 \mathrm{~K}$. The expected absence of dispersion of this value was verified by the ultrasonic pulse transmission technique described in Ref. 16, in the range 1 to $5 \mathrm{MHz}$ as seen in Fig. 1(b), i.e., at frequencies three orders of magnitude below those probed by BLS. Thus, the longitudinal modulus, $M=\rho c_{l}^{2} \approx 1.4 \mathrm{GPa}$, is indeed frequency independent. This value of $M$ is nearly 2000 times the value previously used. The main purpose of this paper

(a)
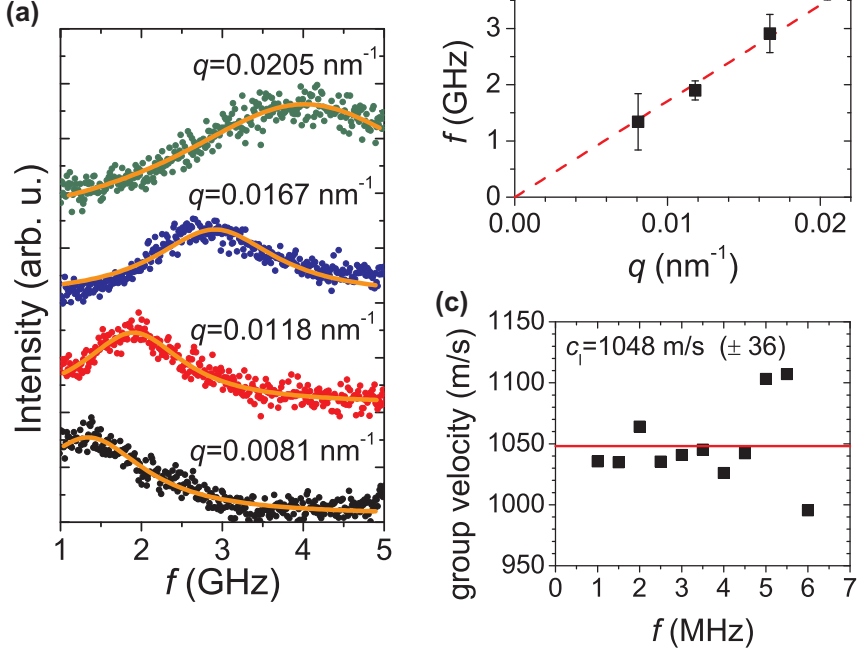

FIG. 1. (Color online) (a) Polarized BLS spectra (anti-Stokes sides only) at different scattering wave vector $q$. (b),(c) The linear dispersion of the longitudinal phonon in the $\mathrm{GHz}$ frequency range (b) and the very similar longitudinal sound velocity in the $\mathrm{MHz}$ regime (c).

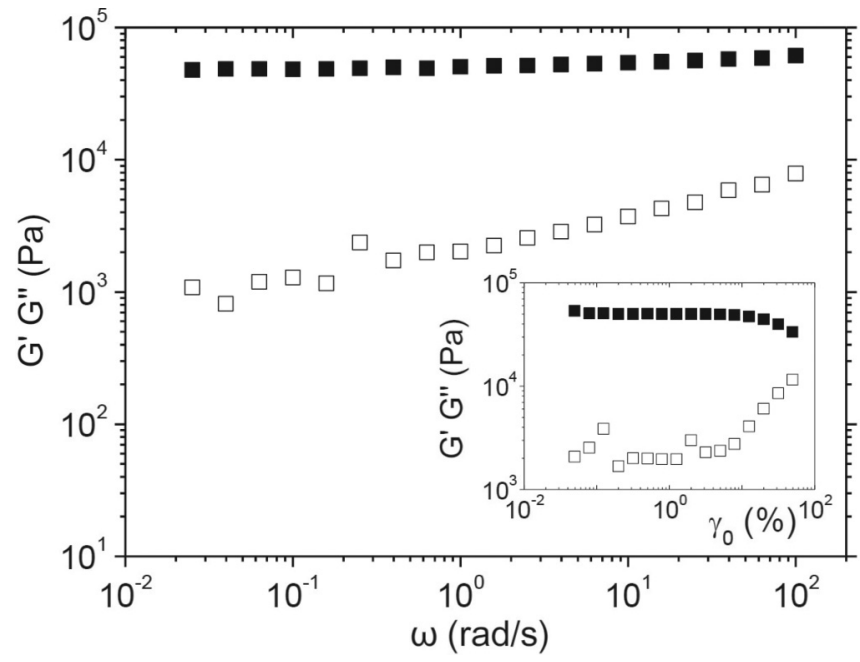

FIG. 2. Frequency-dependent storage $G^{\prime}$ (solid squares) and loss $G^{\prime \prime}$ (open squares) shear moduli of the PDMS rubber in the linear regime (strain amplitude of $1 \%$ ). (Inset) Respective strain sweep data at an oscillation frequency of $1 \mathrm{rad} / \mathrm{s}$.

is to explore the consequence(s) of this altered value of $M$, assuming its value remains at $1.4 \mathrm{GPa}$ down to audio frequencies.

Shear rheometry. For the estimation of $G$, we employed small amplitude oscillatory shear rheometry, which applies a small strain amplitude $\left(\gamma_{0}=1 \%\right)$ in the linear regime. A strain-controlled rheometer (ARES 2kFRTN1 from TA, USA) was utilized with parallel plate geometry (plates from invar, an alloy of copper and iron, with $8 \mathrm{~mm}$ diameter). The sample was placed between the rheometer plates and properly trimmed. Measurements were performed at $25^{\circ} \mathrm{C}$. Applying a sinusoidal strain $\gamma=\gamma_{0} \sin (\omega t)$ with angular frequency $\omega$ led to a stress response $\sigma=\sigma_{0}\left[G^{\prime} \sin (\omega t)+G^{\prime \prime} \cos (\omega t)\right]$. Figure 2 shows the resulting linear response where the storage $\left(G^{\prime}\right)$ and loss $\left(G^{\prime \prime}\right)$ moduli as function of oscillation frequency $(\omega)$ in the range of about 0.01 to $100 \mathrm{rad} / \mathrm{s}$. The inset of Fig. 2 depicts a typical dynamic strain sweep test at a frequency of $1 \mathrm{rad} / \mathrm{s}$, where the regime of strain-independent linear viscoelastic response is observed. Expectedly, this very soft elastomer exhibits (real) $G^{\prime}$ approximately constant in $\omega$, whereas (imaginary) $G^{\prime \prime}$ decreases with decreasing $\omega$ and appears to approach a minimum at the lowest frequencies. The shear modulus is equal to the plateau modulus at low shear rates, $G=G_{\omega \rightarrow 0}^{\prime}=50 \pm 5 \mathrm{kPa}$, in good agreement with the reported shear modulus in Ref. $4(G=40 \mathrm{kPa})$. The low $G$ is indicative of a low degree of cross-linking.

For entangled (not cross linked) PDMS at ambient temperature, i.e., with molecular weight well above the entanglement molecular weight of $12 \mathrm{~kg} / \mathrm{mol}$, the plateau modulus was found to be $\approx 200 \mathrm{kPa} .^{14,17}$ Hence, a low degree of crosslinking is not sufficient to explain the observed low plateau modulus. Some heterogeneity in the sample (e.g., the presence of additives or air bubbles which make the rubber slightly porous without, however, affecting the overall density) could be at the origin this value.

Nanoindentation. We complemented the rheological measurements with nanoindentation using an MFP3D Nano-I (Asymlum Research, USA) equipped with a flat top diamond 


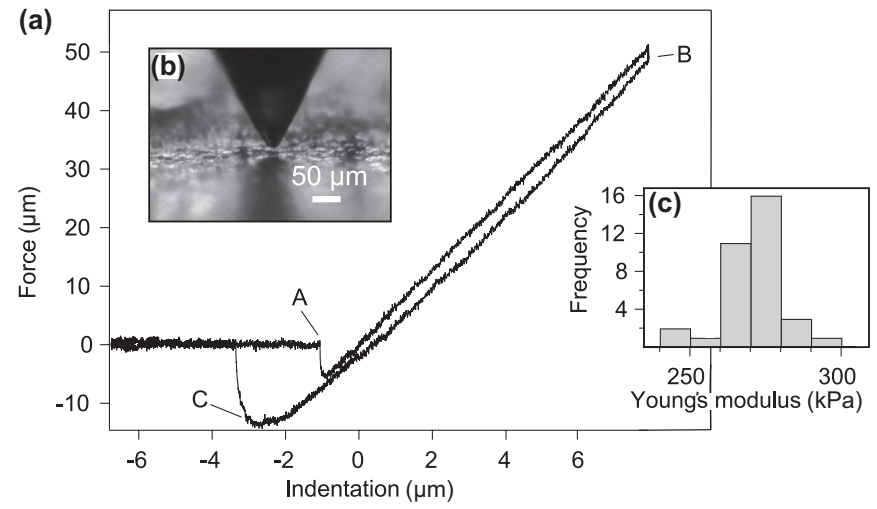

FIG. 3. (a) Nanoindentation curve in the displacement controlled mode with $7 \mu \mathrm{m}$ maximum indentation (less than $5 \%$ of the sample thickness). The curves show a weak snap in upon contact of the indenter tip with the sample (A), small relaxation when a constant indentation depth is held (B), and a clear adhesion (C). (b) The upper inset gives an optical micrograph of the indenter tip while approaching the surface. (c) The retraction curve was fitted with the Oliver and Pharr model between $10 \%$ and $90 \%$ of the maximum load, and the histogram of the measured values of the Young's modulus, $E$.

tip with a diameter of $20 \mu \mathrm{m}$ (Synton-MDP AG, Switzerland). The very small hysteresis of the curve and little drift when holding the force constant (data not shown) are consistent with the small $G^{\prime \prime}$ at low frequencies (Fig. 2). Averaging 36 indentation curves allows the determination of a Young modulus of $E \approx 270 \pm 30 \mathrm{kPa}$.

Notably, this value is not fully consistent with the other experiments. For $M \gg G$, Young's modulus becomes $E \approx$ $3 G$ (cf. Table I); hence, we expect $E \approx 3 \times 50 \mathrm{kPa} \approx 150 \mathrm{kPa}$. However, we measured a Young's modulus of $E=270 \pm$ $30 \mathrm{kPa}$, i.e., almost twice the value expected. Due to the roughness of the sample (see inset to Fig. 3), which was in the same order of the tip size and the indentation depth, the contact area was not well defined. Contact between the sample and the side of the indenter increased the force on the indenter but the corresponding contact area could not be quantified precisely. For this reason, the nanoindentation on this rough surface systematically overestimates $E$.

In any case, we conclude that the measurement of $E$ in rubbery materials is subject to large errors. We therefore speculate that the extremely low value of $M$ (or, in fact, the Lamé coefficient $\lambda$ ) reported in Ref. 4 was indirectly derived from a (potentially erroneous) measurement of $E$ and Poisson's ratio $v$ (see alternative expressions in Table I). Using our experimental moduli, $v=0.49998 \approx 0.5$ because $M \gg G$, whereas the values reported in Ref. 4 lead to $v \approx 0.47$, which is certainly erroneous due to the low value of $M$. (The tabulated value is $v=0.5 .{ }^{14}$ ) Thus, using $\lambda=E v /((1+v)(1-2 v))$ can lead to very large deviations in the derived values of $\lambda$ and, correspondingly, $M$, even if the total value of $v$ was off by a tiny amount. Our value of $M$, on the other hand, is obtained directly from different experiments and is in agreement with the literature value. $^{14}$

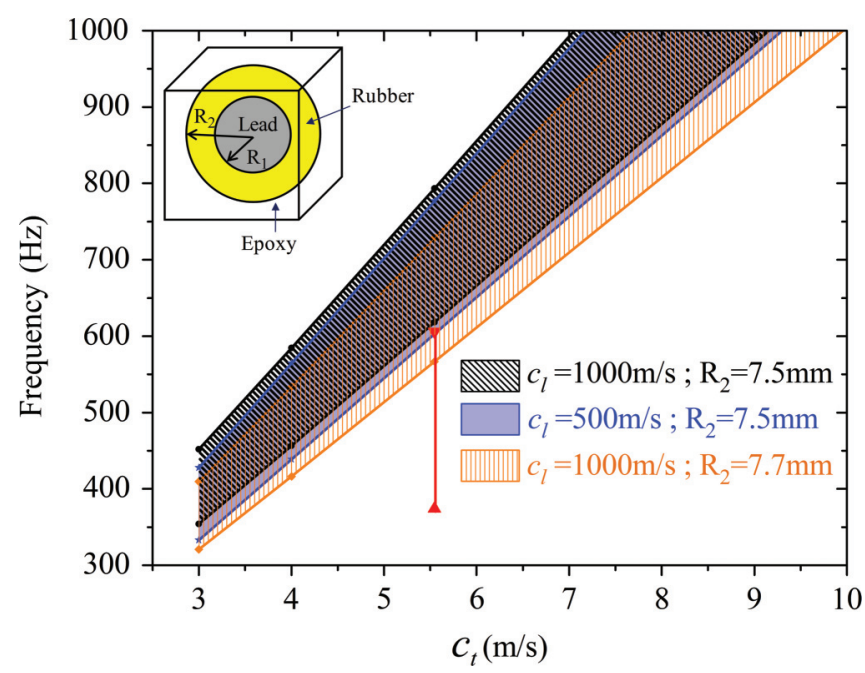

FIG. 4. (Color online) Unit cell of the phononic crystal studied in Ref. 4 (inset). Band gap frequency changes as function of both sound velocities and the thickness of the rubber. Black diagonally hatched and blue solid regions correspond to the gap when $c_{l}$ is fixed to $1000 \mathrm{~m} / \mathrm{s}$ or $500 \mathrm{~m} / \mathrm{s}$, respectively, while the external radius $R_{2}$ is fixed to $7.5 \mathrm{~mm}$. Vertically hatched (orange) regions correspond to the case $R_{2}=7.7 \mathrm{~mm}$ and $c_{l}=1000 \mathrm{~m} / \mathrm{s}$. The red vertical line indicates the gap position for $c_{l}=22.9 \mathrm{~m} / \mathrm{s}$ as used in Ref. 4 .

\section{B. Theoretical calculations}

To study the band gap evolution of the phononic crystal reported in Ref. 4 as function of the longitudinal and shear modulus of the used rubber, band structure calculations were performed using the finite element method. A single unit cell period was used for the simulation with applied Bloch theorem for periodic boundary condition, and the dispersion calculations were performed along the main directions of the first irreducible Brillouin zone as in Ref. 4.

In Fig. 4, we show the band gap frequency for the structure studied in Ref. 4 when using different sets of values for the elastic parameter, expressed in the two velocities, $c_{l}=$ $\sqrt{M / \rho}$ and $c_{t}=\sqrt{G / \rho}$, respectively, but using the correct $c_{l} \approx 1000 \mathrm{~m} / \mathrm{s}$. One can observe that the drop in the gap frequency is related mainly to the decrease of $c_{t}$ and very low frequencies can still be achieved while using the correct values for $c_{l}$. For example, we can still find a gap at $400 \mathrm{~Hz}$ by a structure like in Ref. 4 if we assume values of $c_{t}=3-3.5 \mathrm{~m} / \mathrm{s}$. In fact, changing $c_{l}$ to $500 \mathrm{~m} / \mathrm{s}$ hardly changes the resulting gap frequency by a mere few percent. However, for the value of $c_{t}=5.55 \mathrm{~m} / \mathrm{s}$ used in Ref. 4 (similar to $c_{t} \approx 6.2 \mathrm{~m} / \mathrm{s}$ from our rheometry experiment), the gap is opened above $550 \mathrm{~Hz}$ when $c_{l}$ is in the range $500-1000 \mathrm{~m} / \mathrm{s}$ and, thus, falls quantitatively above the experimentally given value of $400 \mathrm{~Hz}$ unless $c_{l}$ is decreased to about $20 \mathrm{~m} / \mathrm{s}$ like in Ref. 4.

Another parameter which can affect the position of the gap is, for example, the thickness of the rubber layer that can lower the gap when it is increased. For instance, this is shown in Fig. 4 with $c_{l}=1000 \mathrm{~m} / \mathrm{s}$ and $c_{t}=5.55 \mathrm{~m} / \mathrm{s}$, when the external radius $R_{2}$ is increased from 7.5 to $7.7 \mathrm{~mm}$ (which is almost the maximum value of $R_{2}$ before putting the spheres in adjacent unit cells in contact). This shifts the lower limit of 
the gap from 618 to $517 \mathrm{~Hz}$, which is still quantitatively above the value of $400 \mathrm{~Hz}$ obtained in Ref. 4.

Finally, the velocities of sound in the epoxy matrix were also varied by $10 \%$ but we did not find any significant effect on the position and width of the gap. This is physically understandable because the resonances that give rise to the opening of the position gaps are related to the properties of the rubber, and at these low frequencies the "hard materials" lead and epoxy do not undergo deformations but only rigid motions connected by the rubber as a spring.

\section{CONCLUSIONS}

Many simulations in the field of soft phononics employ soft rubber materials with unphysical low longitudinal sound velocity, $c_{l}$, referring to an experimental paper that describes low frequency resonant vibrations in a composite of lead, epoxy, and PDMS rubber. ${ }^{4}$ Applying a combination of different experiments, we measured the elastic moduli of the very same rubber and found values, in particular for $c_{l}$, that are reasonable for such materials. We then showed by band structure calculations that even with such realistic parameters, the low-frequency band gap in Ref. 4 can be reproduced as a result of the low shear modulus found in the material, though the quantitative agreement between theory and experiment is not perfect. These rather small deviations can possibly be attributed to small variations in the (very low) transverse sound velocity or in the structure's geometrical parameters. From an experimentalist's point of view, these results are pleasing and reassuring, and, most probably, some theoretical predictions of peculiar phononic properties based on the originally published extremely low values of sound velocities in rubber still hold by using only a small value of the transverse velocity.

\section{ACKNOWLEDGMENTS}

This work was supported by the Max Planck Society (Germany) and ARISTEIA Program-285 (EU, GSRT-Greece). The work of M.O. and B.D.R. is supported by the Agence Nationale de la Recherche and Direction Générale de l'Armement under the project Metactif, Grant No. ANR-11-ASTR-015.
*Current address: Department of Physics and Astronomy, University of Pennsylvania, Philadelphia, PA 19104, USA.

${ }^{\dagger}$ M. O. and T. S. contributed equally to this work.

${ }^{\ddagger}$ Electronic address: bahram.djafari-rouhani@univ-lille1.fr

§Electronic address: fytas@mpip-mainz.mpg.de

${ }^{1}$ M. M. Sigalas and E. N. Economou, J. Sound Vib. 158, 377 (1992).

${ }^{2}$ M. S. Kushwaha, P. Halevi, L. Dobrzynski, and B. Djafari-Rouhani, Phys. Rev. Lett. 71, 2022 (1993).

${ }^{3}$ M. Maldovan and E. L. Thomas, Periodic Materials and Interference Lithography (Wiley-VCH, New York, 2008).

${ }^{4}$ Z. Y. Liu, X. X. Zhang, Y. W. Mao, Y. Y. Zhu, Z. Y. Yang, C. T. Chan, and P. Sheng, Science 289, 1734 (2000).

${ }^{5}$ T. Still, W. Cheng, M. Retsch, R. Sainidou, J. Wang, U. Jonas, N. Stefanou, and G. Fytas, Phys. Rev. Lett. 100, 194301 (2008).

${ }^{6}$ Z. Yang, J. Mei, M. Yang, N. H. Chan, and P. Sheng, Phys. Rev. Lett. 101, 204301 (2008).

${ }^{7}$ C. J. Naify, C.-M. Chang, G. McKnight, F. Scheulen, and S. Nutt, J. Appl. Phys. 109, 104902 (2011).

${ }^{8}$ L. Bousse, E. Dijkstra, and O. Guenat, Technical Digest, Solid State Sensor and Actuator Workshop, Hilton Head Island, SC, 1996 (Transducers Research Foundation, San Diego, CA, 1996), p. 272.
${ }^{9}$ P. Lambin, A. Khelif, J. O. Vasseur, L. Dobrzynski, and B. Djafari-Rouhani, Phys. Rev. E 63, 066605 (2001).

${ }^{10}$ C. Goffaux, J. Sánchez-Dehesa, A. L. Yeyati, P. Lambin, A. Khelif, J. O. Vasseur, and B. Djafari-Rouhani, Phys. Rev. Lett. 88, 225502 (2002).

${ }^{11}$ J.-C. Hsu and T.-T. Wu, Appl. Phys. Lett. 90, 201904 (2007).

${ }^{12}$ M. Oudich, Y. Li, B. M. Assouar, and Z. Hou, New J. Phys. 12, 083049 (2010).

${ }^{13}$ W. Cheng, J. J. Wang, U. Jonas, G. Fytas, and N. Stefanou, Nat. Mater. 5, 830 (2006).

${ }^{14}$ J. E. Mark, Polymer Data Handbook (Oxford University Press, Oxford, 1999).

${ }^{15} \mathrm{P}$. Voudouris, N. Gomopoulos, A. Le Grand, N. Hadjichristidis, G. Floudas, M. D. Ediger, and G. Fytas, J. Chem. Phys. 132, 074906 (2010).

${ }^{16}$ I. Alig, K. G. Häusler, W. Tanzer, and S. Unger, Acta Polym. 39, 269 (1988).

${ }^{17}$ L. J. Fetters, D. J. Lohse, and R. H. Colby, Physical Properties of Polymers Handbook, edited by J. E. Mark (Springer, Berlin, 2007), Chap. 7, p. 447. 